\title{
The Single Nucleotide Polymorphism rs1014290 of the SLC2A9 Gene Is Associated with Uric Acid Metabolism in Parkinson's Disease
}

\author{
Jiangfang Miao, ${ }^{1,2}$ Jing Liu, ${ }^{1}$ Li Xiao, ${ }^{3}$ Jiedi Zheng, ${ }^{1}$ Chunfeng Liu, ${ }^{1}$ Zufu Zhu, ${ }^{2}$ \\ Kai $\mathrm{Li}^{3}$ and Weifeng Luo ${ }^{1}$ \\ ${ }^{1}$ Jiangsu Key Laboratory of Translational Research and Therapy for Neuro-Psycho-Diseases and The Second Affiliated Hospital of \\ Soochow University, Soochow University, Suzhou, Jiangsu 215021, China \\ ${ }^{2}$ Jiangsu Jiangyin People's Hospital, Jiangyin, Jiangsu 214400, China \\ ${ }^{3}$ College of Pharmaceutical Science, Soochow University, Suzhou, Jiangsu 215123, China
}

Correspondence should be addressed to Kai Li; kaili34@yahoo.com and Weifeng Luo; lwfwxx@126.com

Received 30 May 2017; Revised 14 August 2017; Accepted 22 August 2017; Published 11 October 2017

Academic Editor: Anthony Lang

Copyright (c) 2017 Jiangfang Miao et al. This is an open access article distributed under the Creative Commons Attribution License, which permits unrestricted use, distribution, and reproduction in any medium, provided the original work is properly cited.

Individuals with Parkinson's disease (PD) have lower uric acid levels than those without PD, and the CC genotype and C minor allele of a single nucleotide polymorphism (SNP), rs1014290 of SLC2A9, are associated with lower uric acid levels. We investigated the association of rs1014290 with uric acid metabolism in a cohort of PD cases (220) and controls (110) in a Han Chinese population. Uric acid levels were determined and rs1014290 was assayed using a mutation-sensitive on/off switch technology. PD uric acid levels $(291.65 \pm 76.29 \mu \mathrm{mol} / \mathrm{L})$ were significantly lower than the controls $(325.73 \pm 74.23 \mu \mathrm{mol} / \mathrm{L}, P<0.001$, $t$-test). Individuals with rs1014290 TT and CT genotypes had higher uric acid levels, and those with the CC genotype had the lowest uric acid levels among both control and PD cases. The CC genotype and the C minor allele were statistically more frequent in the PD group compared to the control group. Those with the CC genotype had a statistically significant higher risk of PD than those with the TT or TC genotype (odds ratio $[\mathrm{OR}]=2.249,95 \%$ confidence interval $[\mathrm{CI}]: 1.129-4.480$, and $P=0.021$ ). Thus, SLC2A9 rs1014290 is related to lower uric acid levels in PD patients and can be a risk factor for PD in the Han population.

\section{Introduction}

Parkinson's disease (PD) is one of the most common neurodegenerative diseases, characterized by the extrapyramidal symptoms of resting tremor, rigidity, bradykinesia, and postural instability. Males have a significantly higher incidence rate of $\mathrm{PD}$, approximately 1.5 times greater than females [1]. Although the etiology of PD remains unclear, it is generally considered to be a combination of complex genetic and environmental factors [2]. Several epidemiological studies suggest that high plasma uric acid levels decrease the risk of developing PD $[3,4]$, and serum uric acid can be a predictor of clinical and radiographic progression in $\mathrm{PD}$ [5].

$S L C 2 A 9$ is considered to be the most effective urate transporter [6], affecting circulating uric acid levels. The single nucleotide polymorphism (SNP) rs1014290 of the SLC2A9 gene has been reported to influence the age of onset of $\mathrm{PD}$ [7].
Here, through a retrospective case-controlled study, we aimed to identify the correlation between SLC2A9 SNP rs1014290 and $\mathrm{PD}$ pathogenesis and to explore whether this relationship was affected by uric acid metabolism in the Han Chinese population. In all, we aimed to find genotype-endophenotypeexophenotype relationships among rs1014290 in SLC2A9, serum uric acid level, and PD in a select Han Chinese subpopulation.

\section{Methods}

2.1. Participants. A total of 220 patients with sporadic idiopathic PD from the Neurology Department, Second Affiliated Hospital of Soochow University in China, were selected from January 2011 to November 2012. The PD diagnosis was made by neurologists based on criteria consistent with the UK PD Society (UKPDS) Brain Bank criteria [8]. Sporadic idiopathic 


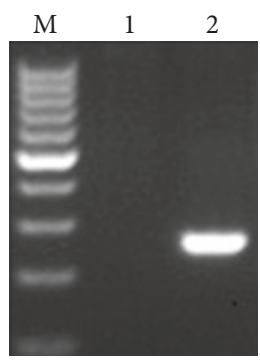

(a)

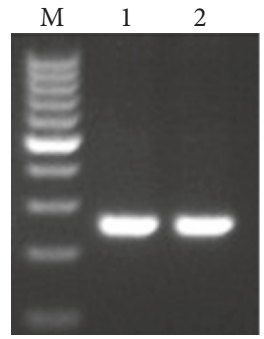

(c)

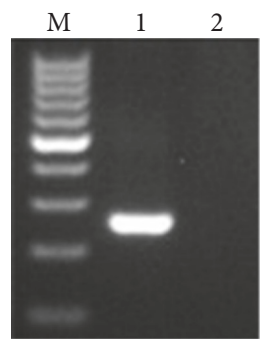

(e)

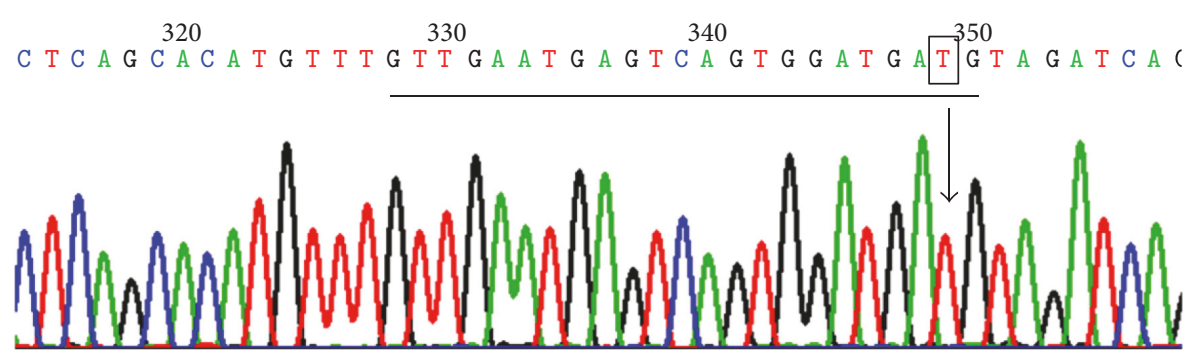

(b)
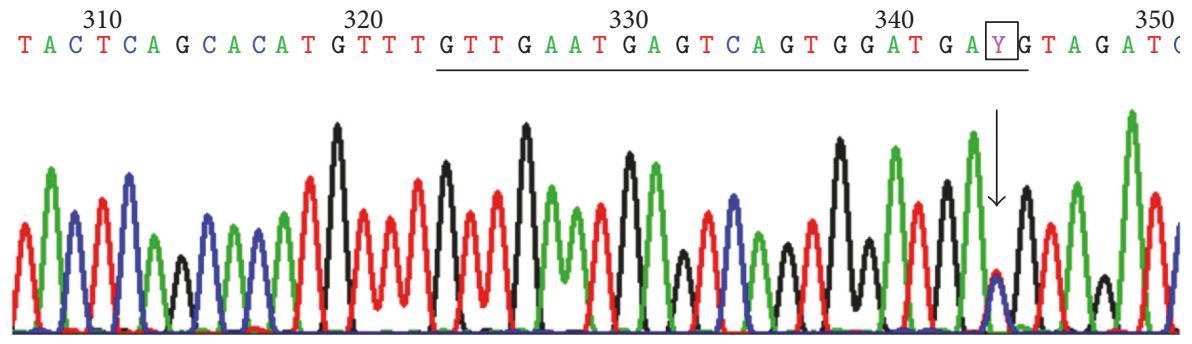

(d)

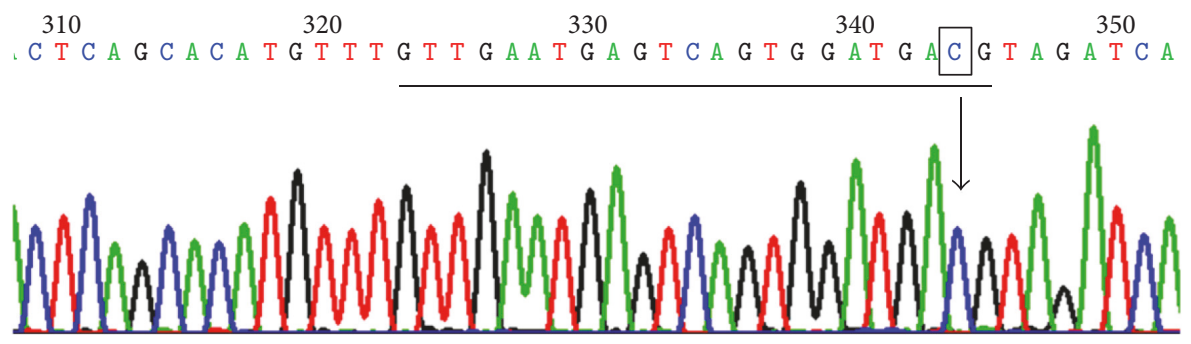

(f)

FIGURE 1: Representative illustrations of genotyping data and sequencing chromatography results. PCR product from the C allele template (lane 1) and the amplified fragment from the T allele-specific DNA using the on/off switch (lane 2). (a, c, and e) are the relevant genotypes confirmed by Sanger sequencing. The corresponding genotypes are CC, CT (Y), and TT as indicated with the box. M, marker.

PD is defined as one without a family history of PD. For comparison, 110 unrelated Han healthy controls matched in gender, age, and area of residence were included and investigated by our research group during the PD epidemiological study. All healthy controls were examined by neurologists to rule out neurodegenerative disorders, and detailed clinical data was recorded. And those who had gout and other diseases, such as liver and kidney diseases, were ruled out in all subjects. All subjects provided written informed consent. Ethics approval was obtained from the ethics committee of the Second Affiliated Hospital of Soochow University in China. $4 \mathrm{ml}$ of venous blood was extracted from all subjects in the fasting state, $2 \mathrm{ml}$ for testing serum uric acid levels and $2 \mathrm{ml}$ for gene detection.

2.2. Uric Acid Levels Testing. Uric acid levels were determined using a Beckman Coulter (USA) automatic biochemical analyzer with the original kits at the Second Affiliated Hospital of Soochow University.

2.3. Genotyping. DNA was extracted from peripheral blood using the Magman TM Blood DNA Kit (GenoTheramics, USA) based on magnetic beads. Genotyping for rs1014290 was performed using a new assay employing proofreading PCR technology. We assayed SNP genotyping using a mutation-sensitive on/off switch consisting of $3^{\prime}$ terminal phosphorothioate-modified allele-specific primers and highfidelity exo+ DNA polymerases. In recent years, this novel technology has been successfully employed in genetic analysis [9-11]. The mutation-sensitive on/off switch eliminates false positive errors and significantly decreases false negatives, which has been demonstrated to have a higher specificity than assays mediated by low-fidelity DNA polymerase. During the assay development stage, Sanger sequencing was used to confirm the proofreading PCR results (Figure 1).

2.4. Statistical Analyses. All statistical analyses were performed using IBM SPSS Statistics 23. The Hardy-Weinberg equilibrium (HWE) of SLC2A9 rs1014290 for PD patients and controls was verified by Pearson's Chi-Square test, and "deviation" from Hardy-Weinberg is being tested. Allele frequency and genotype frequency between the two groups were compared with Chi-Square test. Uric acid levels were used to compare between the two groups using independent sample $t$-test. The odds ratio (OR) and $95 \%$ confidence interval (CI) were estimated using Chi-Square tests to examine the 
TABLE 1: Clinical characteristics of study participants. The mean onset age of PD patients with the rs1014290 CC genotype was lower than that of those with the TT + TC genotype.

\begin{tabular}{lccccc}
\hline \multirow{2}{*}{ Group } & \multicolumn{2}{c}{ PD } & \multirow{2}{*}{ Controls } & \multicolumn{2}{c}{ C } \\
& CC & TT + CT & & $17(8 / 9)$ & $93(52 / 41)$ \\
Number (M/F) & $66(37 / 29)$ & $154(84 / 70)$ & & $69.59 \pm 4.93$ & $69.46 \pm 8.72$ \\
Age (years) & $65.48 \pm 9.62$ & $69.22 \pm 9.33$ & 0.009 & 0.933 \\
Age at onset (years) & $59.24 \pm 9.77$ & $63.21 \pm 9.51$ & 0.005 & & \\
Course of disease & $6.24 \pm 4.00$ & $6.01 \pm 4.05$ & 0.699 & & \\
UPDRS motor scale & $23.21 \pm 10.82$ & $23.12 \pm 10.88$ & 0.956 & & \\
H-Y score & $2.00 \pm 0.81$ & $2.05 \pm 0.86$ & 0.714 & & \\
\hline
\end{tabular}

TABLE 2: Frequency of rs1014290 in PD patients and controls in a Han subpopulation. The frequency of the natural CC genotype is significantly higher in PD patients as compared to the controls, and less TT and CT genotypes are observed in PD patients. Analysis of C and T alleles revealed significant differences between groups.

\begin{tabular}{|c|c|c|c|c|c|c|c|c|}
\hline \multirow{2}{*}{ Group } & \multirow{2}{*}{ Number } & \multicolumn{3}{|c|}{ Genotype frequency (\%) } & \multirow{2}{*}{$P$} & \multicolumn{2}{|c|}{ Allele frequency (\%) } & \multirow{2}{*}{$P$} \\
\hline & & TT & CT & $\mathrm{CC}$ & & $\mathrm{T}$ & $\mathrm{C}$ & \\
\hline $\mathrm{PD}$ & 220 & $56(25.5)$ & $98(44.5)$ & $66(30.0)$ & \multirow{2}{*}{$<0.001$} & $210(47.7)$ & $230(52.3)$ & \multirow{2}{*}{$<0.001$} \\
\hline Controls & 110 & $33(30.0)$ & $60(54.5)$ & $17(15.5)$ & & $126(57.3)$ & $94(42.7)$ & \\
\hline
\end{tabular}

CC genotype frequency and minor allele frequencies for rs1014290 between PD patients and controls.

\section{Results}

3.1. Clinical Characteristics of Study Participants. This study included 220 patients with idiopathic PD and 110 unrelated controls, of which $54.5 \%$ were male. There was no difference between the mean ages of PD patients (68.10 \pm 9.55 years) and those of controls $(69.48 \pm 8.23$ years, $P=0.175, t$-test $)$. The mean serum uric acid concentration of PD patients $(291.65 \pm$ $76.29 \mu \mathrm{mol} / \mathrm{L})$ tended to be lower than that of the controls $(325.73 \pm 74.23 \mu \mathrm{mol} / \mathrm{L}, P<0.001$, $t$-test) (Table 1$)$.

3.2. The rs1014290 C Allele Genotype Was More Prevalent in the PD Group Compared to the Control Group. The genotype frequencies of the PD group and the control group were in agreement with the HWE (PD: $\chi^{2}=1.318, P>0.05$; controls: $\chi^{2}=0.689, P>0.05$, Pearson Chi-Square test). The minor allele frequency of rs1014290 was $52.0 \%$ for the PD group and $42.7 \%$ for the control group, which were statistically significant $\left(\chi^{2}=5.347, P=0.021\right.$, OR $=0.681$, and 95\% CI $=0.492-0.944$, Pearson Chi-Square test) (Table 2). The CC genotype frequencies of rs 1014290 were $30.0 \%$ for the PD group and $15.5 \%$ for the control group $\left(\chi^{2}=8.242\right.$, $P=0.004, \mathrm{OR}=0.427$, and $95 \% \mathrm{CI}=0.236-0.771$, Chi-Square test) (Table 2). We then performed multiple factors logistic regression analysis of rs1014290 genotypes on PD risk and found that individuals with the CC genotype had a higher risk of $\mathrm{PD}$ than individuals with the TT or TC genotype (OR $=2.189,95 \% \mathrm{CI}=1.095-4.376$, and $P=0.027$ ) (Table 3 ).

3.3. Clinical Characteristics of PD Patients. The mean onset age of PD patients with the rs1014290 CC genotype (59.24 \pm 9.77 years) was lower than that of those with the TT + TC genotype $(63.21 \pm 9.51$ years, $P=0.005, t$-test $)$. For detailed data of clinical characteristics of PD patients please see Table 1.

3.4. Genotype-Dependent Serum Uric Acid Levels. The mean serum uric acid concentration of individuals with the CC genotype was lower than that of those with the TT and CT genotypes in both the control $(P<0.001, t$-test $)$ and $\mathrm{PD}$ $(P<0.001, t$-test) groups (Table 4$)$.

\section{Discussion}

Uric acid is a strong antioxidant in the human body, having a protective effect against oxidative stress and free radical damage, and may slow the progression of many degenerative nervous diseases [12-14]. Uric acid levels in the brain spinal fluid have a linear relationship with levels found in the blood. The protective effect of high levels of uric acid was first reported by Simon and Vanvunakis in 1964 [15]. Thereafter, several epidemiological studies found that high serum uric acid levels were associated with later onset of PD in males or alleviation of the severity of PD in recent years [4, 16, 17]. Genetic engineering technology has been used to dissect the relationship between uric acid levels in the brain and the development of PD [18], and similar data was gleaned from autopsies performed on patients with PD [19]. As all causal genes dissected from PD genetics account for only approximately $5 \%$ of PD cases with familial inheritance, confirmation of the large number of modulation genes of PD has several clinical impacts.

SLC2A9 is the most effective one of all uric acid transporters, and SLC2A9 variants have been shown to influence circulating uric acid levels. SLC2A9's involvement in uric acid metabolism has been documented by both clinical and animal studies $[6,20,21]$. It is proved that the SLC2A9 variant rs3733591 served as an important genetic checkpoint for tophaceous gout and increased uric acid levels from two geographically diverse populations [22]. Various mutations of 
TABLE 3: Multiple factors logistic regression analysis of the rs1014290 genotype in SLC2A9 on the risk of PD*. Individuals with the CC genotype in SLC2A9 had a higher risk of PD than individuals with the TT or TC genotype.

\begin{tabular}{ccccccrrr}
\hline & $B$ & SE & Wald & $v$ & $P$ & OR & Lower \\
\hline TT & & & 6.579 & 2 & 0.037 & & \\
TC & -0.006 & 0.274 & 0.000 & 1 & 0.983 & 0.994 & 0.581 \\
CC & 0.783 & 0.354 & 4.909 & 1 & 0.027 & 2.189 & 1.095 \\
\hline
\end{tabular}

*Adjusted for age, sex, height, and body weight.

TABLE 4: Average serum uric acid levels in the PD and control groups. Individuals and PD patients carrying the CC genotype had lower uric acid levels as compared to those with the TT and CT genotypes.

\begin{tabular}{lcccc}
\hline Group & Number & & UA $(\mu \mathrm{mol} / \mathrm{L})$ & CC \\
\hline PD & & TT + CT & $264.00 \pm 45.22$ & $<0.001$ \\
Controls & 220 & $303.50 \pm 83.62$ & $279.71 \pm 18.32$ \\
& $P$ & 110 & $334.14 \pm 77.50$ & 0.031 \\
\hline
\end{tabular}

$P$ values compare individuals with different genotypes within the same group; UA, uric acid.

SLC2A9 were found in hypouricemia patients: Ile118HisfsX27 in a Czech family [23], exon 7 deletion in Ashkenazi Jewish [24], and R380W and R198C in Japanese [25]. Among the large number of genes related to uric acid metabolism, the present study clearly illustrated the close relationship between rs1014290 genotypes in SLC2A9 and serum uric acid levels; individuals with the TT and CT genotypes have higher uric acid levels, and those with the CC genotype have the lowest uric acid level. This strong relationship between rs1014290 genotypes in SLC2A9 and uric acid level phenotype was observed in the normal controls of our present study. Furthermore, we found that PD patients with TT and CT genotypes had higher uric acid levels than those with the CC genotype. The genotype-endophenotype relationship of rs1014290 to the serum uric acid level was also observed in the PD group. Our present study demonstrated that the mean serum uric acid concentration of PD patients was lower than that of the controls, which is consistent with our previous study [26]. We found that, compared with the control group, the rs1014290 minor allele and CC genotype frequencies were higher in the PD group. Furthermore, multiple factors logistic regression analysis showed that individuals with the CC genotype had a statistically significant higher risk of PD than individuals with the TT or TC genotype. The present study has established a link between rs1014290 and PD in a relatively large group of Han Chinese. We suggest that this PD phenotype related to the rs1014290 genotype is possibly mediated through the endophenotype of uric acid metabolism.

The mean onset age of PD patients with the CC genotype was relatively low, which confirms that the CC genotype influences the age of onset of PD [7]. Therefore, there is a genotype-phenotype association of SLC2A9 rs1014290 with PD through uric acid metabolism in Han Chinese. The polymorphism of rs1014290 in SLC2A9 may be a risk factor for PD in the Han Chinese population.

Uric acid levels of PD patients with TT and CT genotypes were significantly reduced compared to the controls. Low serum uric acid levels in PD patients were associated not only with the polymorphism of rs1014290 in SLC2A9 loci, but also with other factors. Various SNPs near SLC2A9 have shown to be linked to uric acid level, such as SLC17A1, SLC22A11, and SLC22A12 [27].

Based upon these data, we suggest that SLC2A9 can be used as either a new drug target or a target for gene therapy. In addition to the potential application in the development of new therapies for PD, the confirmed relationship between rs1014290 and PD may also have diagnostic implications. As rs1014290 in SLC2A9 is only a modulation factor, its value in the diagnosis and prediction of relative risk to a particular individual may be limited. However, when genotyping is combined with measurement of serum uric acid, high risk or susceptible individuals could be identified. In fact, by further including family history, a more informed assessment could be provided. And the study sample of our study was relatively small; we should collect more samples for further analysis, as larger sample sizes would have allowed further subanalyses and conditional analysis of SNPs in moderate-to-high link with rs1014290 near SLC2A9.

\section{Conclusions and Future Directions}

In conclusion, the present study bridged the divide between genotype, endophenotype, and exophenotype through analysis of rs1014290, serum uric acid level, and incidence of PD. We further confirmed that relatively low uric acid level is a risk factor for $\mathrm{PD}$ pathogenesis and that the rs1014290 of SLC2A9 can be a risk factor for PD in the Han population. The protective effect of high serum uric acid level has been reinforced by our study. As the genotyping assay developed by this study is simple, cost-effective, and extremely reliable, its wide application may be beneficial to $\mathrm{PD}$ patients for screening purposes as well as individualized medicine. 


\section{Conflicts of Interest}

The authors declare that there are no conflicts of interest regarding the publication of this article.

\section{Authors' Contributions}

Jiangfang Miao and Jing Liu contributed equally to this work.

\section{Acknowledgments}

The authors would like to thank Collin Yan for assistance in proofreading this manuscript. This work was supported by Jiangsu Key Laboratory of Translational Research and Therapy for Neuro-Psycho-Diseases (BM2013003), a project funded by the Priority Academic Program Development of Jiangsu Higher Education Institutions, Jiangsu Provincial Special Program of Medical Science (BL2014042), the Guide Project for Suzhou Science and Technology Development Plan (SYSD2014088), the Youth Science and Technology Project of the Health Bureau of Suzhou City (KJXW2014012), the Pre-Research Foundation of the Second Affiliated Hospital of Soochow University (SDFEYQN1407), Suzhou Clinical Key Disease Diagnosis and Treatment Technology Foundation (LCZX201304), the Key Project of Science and Technology Innovation of Soochow University (SZ123819), Suzhou Technology Support Project (SS201112), and the Chinese National 863 Major Grant (no. 2012AA020905).

\section{References}

[1] G. F. Wooten, L. J. Currie, V. E. Bovbjerg, J. K. Lee, and J. Patrie, "Are men at greater risk for Parkinson's disease than women?" Journal of Neurology, Neurosurgery \& Psychiatry, vol. 75, no. 4, pp. 637-639, 2004.

[2] J. L. Eriksen, Z. Wszolek, and L. Petrucelli, "Molecular pathogenesis of Parkinson disease," Archives of Neurology, vol. 62, no. 3, pp. 353-357, 2005.

[3] M. K. Kutzing and B. L. Firestein, "Altered uric acid levels and disease states," Journal of Pharmacology and Experimental Therapeutics, vol. 324, no. 1, pp. 1-7, 2008.

[4] A. Winquist, K. Steenland, and A. Shankar, "Higher serum uric acid associated with decreased Parkinson's disease prevalence in a large community-based survey," Movement Disorders, vol. 25, no. 7, pp. 932-936, 2010.

[5] M. A. Schwarzschild, S. R. Schwid, K. Marek et al., "Serum urate as a predictor of clinical and radiographic progression in Parkinson disease," Archives of Neurology, vol. 65, no. 6, pp. 716723, 2008.

[6] M. J. Caulfield, P. B. Munroe, D. O’Neill et al., "SLC2A9 is a highcapacity urate transporter in humans," PLoS Medicine, vol. 5, no. 10, article e197, pp. 1509-1523, 2008.

[7] M. F. Facheris, A. A. Hicks, C. Minelli et al., "Variation in the uric acid transporter gene SLC2A9 and its association with AAO of Parkinson's disease," Journal of Molecular Neuroscience, vol. 43, no. 3, pp. 246-250, 2011.

[8] A. J. Hughes, S. E. Daniel, L. Kilford, and A. J. Lees, "Accuracy of clinical diagnosis of idiopathic Parkinson's disease: a clinicopathological study of 100 cases," Journal of Neurology Neurosurgery and Psychiatry, vol. 55, no. 3, pp. 181-184, 1992.
[9] J. Zhang, K. Li, J. R. Pardinas, S. S. Sommer, and K.-T. Yao, "Proofreading genotyping assays mediated by high fidelity exo+ DNA polymerases," Trends in Biotechnology, vol. 23, no. 2, pp. 92-96, 2005.

[10] J. Zhang, K. Li, J. R. Pardinas, D. F. Liao, H. J. Li, and X. Zhang, "SNP discrimination through proofreading and OFF-switch of Exo+ polymerase," Applied Biochemistry and Biotechnology Part B: Molecular Biotechnology, vol. 27, no. 1, pp. 75-80, 2004.

[11] L. J. Reha-Krantz, "DNA polymerase proofreading: multiple roles maintain genome stability," Biochimica et Biophysica Acta-Proteins and Proteomics, vol. 1804, no. 5, pp. 1049-1063, 2010.

[12] M. Rentzos, C. Nikolaou, M. Anagnostouli et al., "Serum uric acid and multiple sclerosis," Clinical Neurology and Neurosurgery, vol. 108, no. 6, pp. 527-531, 2006.

[13] T.-S. Kim, C.-U. Pae, S.-J. Yoon et al., "Decreased plasma antioxidants in patients with Alzheimer's disease," International Journal of Geriatric Psychiatry, vol. 21, no. 4, pp. 344-348, 2006.

[14] I. Schlesinger and N. Schlesinger, "Uric acid in Parkinson's disease," Movement Disorders, vol. 23, no. 12, pp. 1653-1657, 2008.

[15] M. I. Simon and H. Vanvunakis, "The dye-sensitized photooxidation of purine and pyrimidine derivatives," Archives of Biochemistry and Biophysics, vol. 105, no. 1, pp. 197-206, 1964.

[16] T. Annanmaki, A. Muuronen, and K. Murros, "Low plasma uric acid level in Parkinson's disease," Movement Disorders, vol. 22, no. 8, pp. 1133-1137, 2007.

[17] M. G. Weisskopf, E. O’Reilly, H. Chen, M. A. Schwarzschild, and A. Ascherio, "Plasma urate and risk of Parkinson's disease," American Journal of Epidemiology, vol. 166, no. 5, pp. 561-567, 2007.

[18] R. F. Anderson and T. A. Harris, "Dopamine and uric acid act as antioxidants in the repair of DNA radicals: implications in Parkinson's disease," Free Radical Research, vol. 37, no. 10, pp. 1131-1136, 2003.

[19] W. H. Church and V. L. Ward, "Uric acid is reduced in the substantia nigra in Parkinson's disease: effect on dopamine oxidation," Brain Research Bulletin, vol. 33, no. 4, pp. 419-425, 1994.

[20] A. Döring, C. Gieger, D. Mehta et al., "SLC2A9 influences uric acid concentrations with pronounced sex-specific effects," Nature Genetics, vol. 40, no. 4, pp. 430-436, 2008.

[21] V. Vitart, I. Rudan, C. Hayward et al., "SLC2A9 is a newly identified urate transporter influencing serum urate concentration, urate excretion and gout," Nature Genetics, vol. 40, no. 4, pp. 437-442, 2008.

[22] H.-P. Tu, C.-J. Chen, S. Tovosia et al., "Associations of a nonsynonymous variant in SLC2A9 with gouty arthritis and uric acid levels in Han Chinese subjects and Solomon Islanders," Annals of the Rheumatic Diseases, vol. 69, no. 5, pp. 887-890, 2010.

[23] B. Stiburkova, K. Ichida, and I. Sebesta, "Novel homozygous insertion in SLC2A9 gene caused renal hypouricemia," Molecular Genetics and Metabolism, vol. 102, no. 4, pp. 430-435, 2011.

[24] D. Dinour, N. K. Gray, S. Campbell et al., "Homozygous SLC2A9 mutations cause severe renal hypouricemia," Journal of the American Society of Nephrology, vol. 21, no. 1, pp. 64-72, 2010.

[25] H. Matsuo, T. Chiba, S. Nagamori et al., "Mutations in Glucose Transporter 9 Gene SLC2A9 Cause Renal Hypouricemia," American Journal of Human Genetics, vol. 83, no. 6, pp. 744-751, 2008. 
[26] X. J. Wang, W. F. Luo, L. J. Wang et al., "Study on uric acid and the related factors associated with cognition in the patients with Parkinson's disease," Zhonghua Yi Xue Za Zhi, vol. 89, no. 23, pp. 1633-1635, 2009.

[27] M. Kolz, T. Johnson, S. Sanna et al., "Meta-analysis of 28,141 individuals identifies common variants within five new loci that influence uric acid concentrations," PLoS Genetics, vol. 5, no. 6, Article ID e1000504, 2009. 


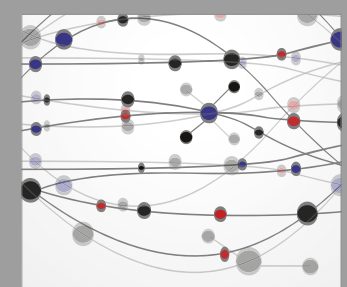

The Scientific World Journal
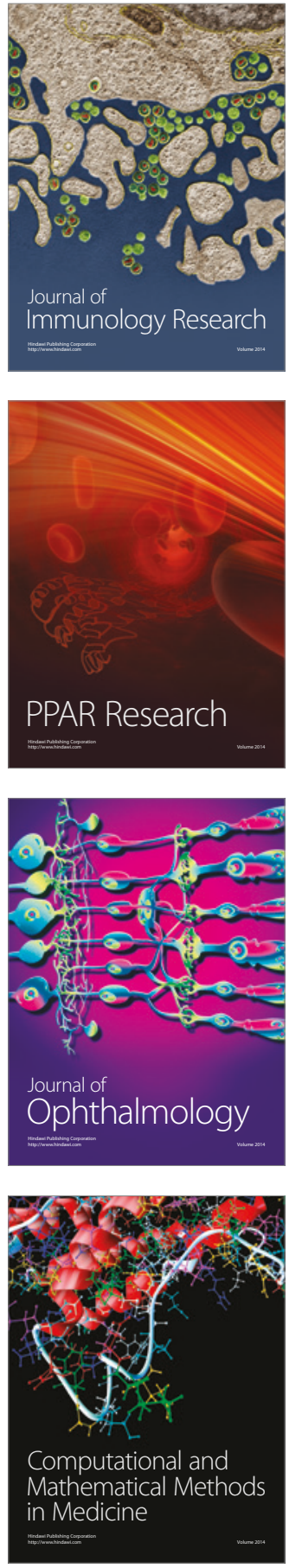

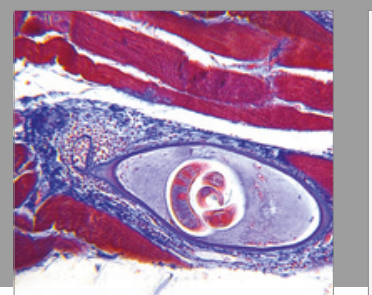

Gastroenterology Research and Practice
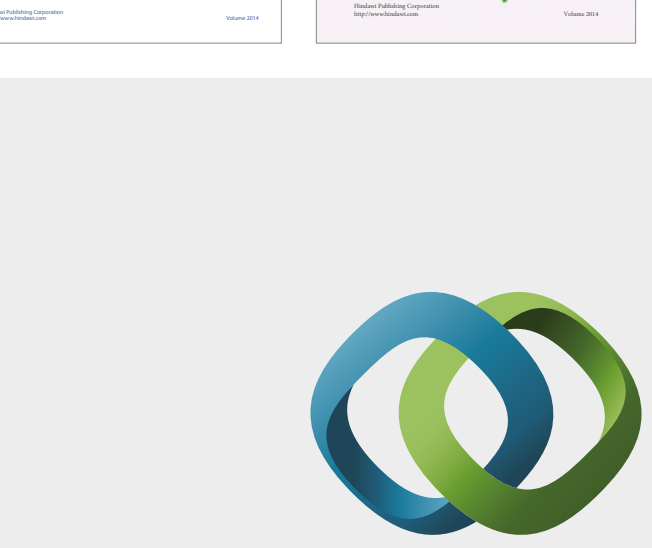

\section{Hindawi}

Submit your manuscripts at

https://www.hindawi.com
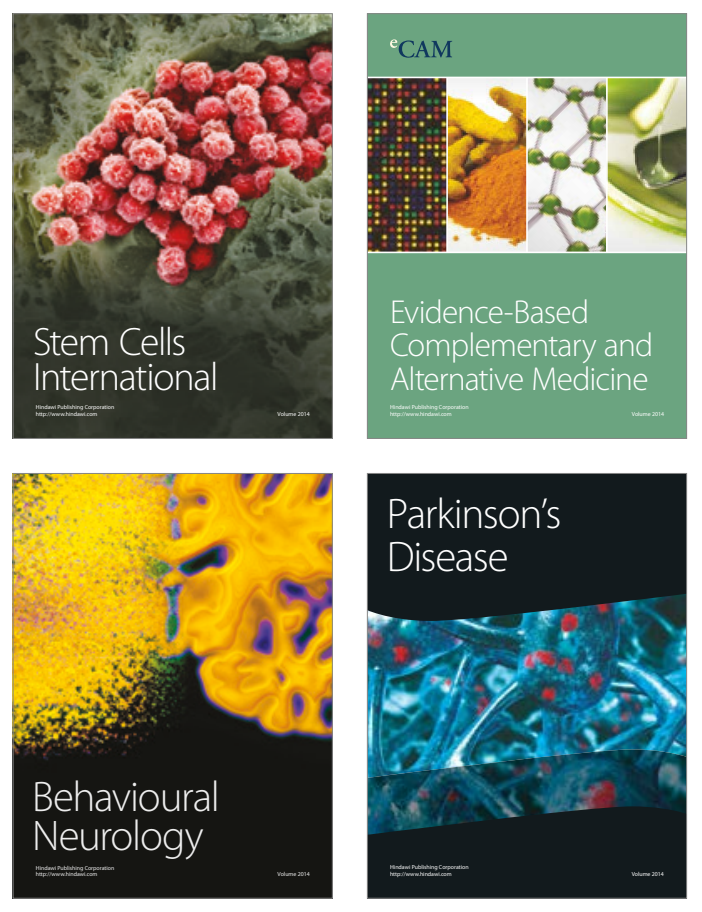
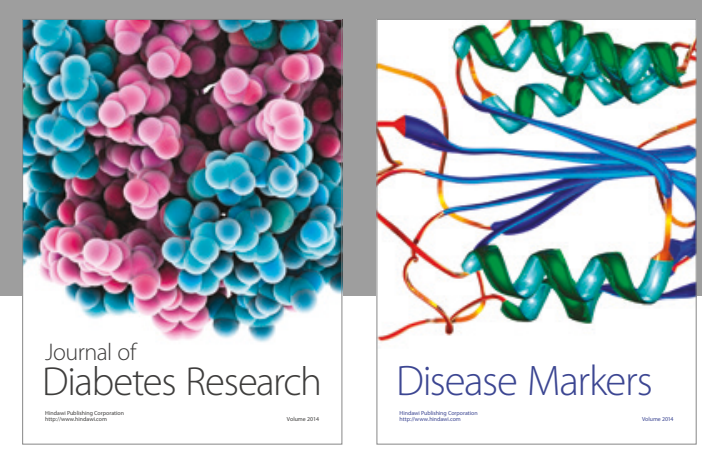

Disease Markers
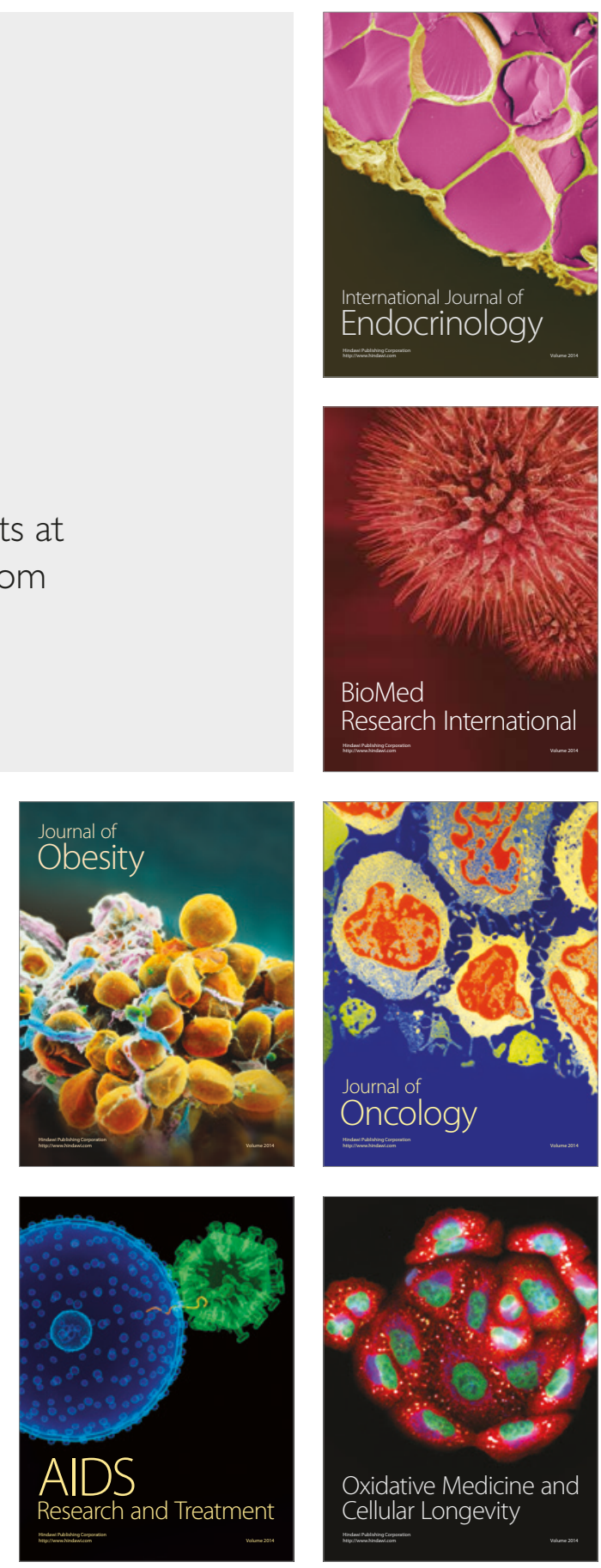